\title{
.
}

Rafael Linde García*

\section{LA ESTRATEGIA DE CHINA EN EL NUEVO ORDEN ECONÓMICO MUNDIAL}

En el presente artículo se analizan las relaciones económicas y comerciales de China con el resto del mundo y su estrategia en el nuevo orden económico mundial. En particular, se examina el papel de China en las relaciones económicas y comerciales contemporáneas, desafíos y obstáculos a los que se enfrenta, y las prioridades del país asiático dentro del necesario equilibrio entre el mantenimiento de la paz social en el interior del país ${ }^{1}$ y aumentar su peso e influencia en el exterior.

Palabras clave: innovación, apertura, cooperación, dominio, foro.

Clasificación JEL: D78, O31, O40.

\section{Introducción}

El ascenso de China como potencia económica mundial de primer orden en los últimos años es algo indiscutible. Unido a este ascenso existe un debate sobre las verdaderas intenciones de China en su plan de expansión internacional, en las relaciones con sus principales socios comerciales y en la arquitectura financiera mundial. Este debate ha sido especialmente intenso en los últimos años, en los que muchas empresas chinas han accedido a empresas y sectores estratégicos de países occidentales, aprovechando las bajas cotizaciones de algunos activos por la crisis económica y financiera. Además, la aparición de nuevas

*Técnico Comercial y Economista del Estado. Versión de enero de 2018.

1 La brecha de renta y cultural entre el campo y la ciudad es muy grande en China (aunque el coeficiente de Gini, que mide la desigualdad de la renta, viene mejorando desde 2008), y todavía el 43 por 100 de la población vive en zonas rurales. instituciones e iniciativas de carácter multilateral o trasnacional justifica analizar el contexto actual y los principales intereses del país asiático. El análisis nos hará concluir que China no pretende un cambio radical del orden económico internacional. Puede decirse que sus principales intereses son el mantenimiento de la paz social interna, en un contexto de cambio de patrón de crecimiento (lo cual asegura que este cambio sea muy gradual y controlado), la hegemonía política en la zona de su influencia (Asia Oriental y mar Meridional) y la adquisición de la tecnología y know how claves para dar el «salto» en la escala tecnológica de producción.

\section{Situación de China en el contexto económico y comercial internacional}

La emergencia de China como potencia económica global ha sido uno de los casos $\triangleright$ 
más relevantes desde el final de la Guerra Fría. Según datos del Fondo Monetario Internacional, China es la segunda mayor economía del mundo (solo por detrás de Estados Unidos), con un PIB en 2016 de 11,2 billones de dólares. A pesar de la desaceleración económica que está sufriendo en los últimos años (crecimiento del 6,7 por 100 del PIB en 2016, la menor tasa de crecimiento en 26 años), muchos analistas prevén que China desbanque a Estados Unidos como primera economía mundial antes del año 2030. China cuenta con unas bases sólidas para que sea así: aparte de que se trata del país más poblado del mundo (1.382 millones de habitantes en 2016), es el mayor consumidor de energía, el mayor tenedor de reservas exteriores del mundo (3,05 billones de dólares en 2016), el primer socio comercial para una larga lista de países del mundo y el mayor mercado mundial para infinidad de productos, como los automóviles.

Todos estos hechos son el resultado de una tendencia de casi cuarenta años en los que China ha seguido un «patrón de crecimiento asiático» (como ya hicieran Japón o Corea del Sur), que consiste en confiar la modernización de la economía al desarrollo del sector exportador. Se trata de una economía muy orientada a la industria y a la inversión, con fuerte dependencia del crédito y un apoyo decidido a las empresas públicas.

Como es lógico, esto se ha traducido en una mayor aportación de China al crecimiento económico global. Si en la década de los ochenta su aportación al crecimiento global era de apenas un 1,6 por 100, hoy contribuye en más de una quinta parte. Las vías por las que se produce esta contribución son varias: en China se localizan importantes centros financieros (como Shanghai o Pekín), con gran impacto en los mercados bursátiles del resto del mundo (como se vio durante las turbulencias bursátiles de 2015 y 2016); es un mercado enorme para las exportaciones (y las importaciones) de numerosos países; está llamado a ser una gran potencia turística, dada la gran población que tiene y el aumento gradual del turismo chino en el exterior; dados sus bajos costes de producción, es un país que ha exportado desinflación al resto del mundo y aumentado su bienestar (al abaratar los costes de producción para las empresas que fabrican en China o importan de ese país, se produce un aumento de las posibilidades de producción a escala global); incluso se puede decir que China ha contribuido a la estabilidad económica durante la crisis económica y financiera mundial: durante esos años el crecimiento de la economía china siguió impulsado por una decidida política de demanda (especialmente por inversión en infraestructuras) ${ }^{2}$. De esta manera, China ha pasado de afectar únicamente en los países de su entorno geográfico a ser un actor decisivo en la economía mundial, y de proporcionar inestabilidad y pobreza en el mundo a reducir significativamente el nivel de pobreza en el mundo. Sin embargo, la economía china afronta en la actualidad una serie de desafíos importantes y debilidades estructurales que exigen una respuesta decisiva de las autoridades. Nos referimos, por ejemplo, al progresivo envejecimiento de su población, a la inexistencia de un sistema legal independiente, a la excesiva dependencia energética o al propio modelo de crecimiento. Este modelo de crecimiento ha provocado una sobrevaloración de activos (como acciones y viviendas) y la sobreproducción en algunos casos que, unidos a la caída de la demanda global con la crisis, ha derivado en exceso de capacidad en algunos sectores. $D$

\footnotetext{
2 Por ejemplo, Pekín aprobó un paquete de estímulo de 415.000 millones de euros y se concedieron créditos por valor de un billón de euros. Además, China ha participado activamente en las ayudas y préstamos bilaterales para ayudar a países en dificultades.
} 
Para el primer caso, basta recordar las turbulencias en los mercados bursátiles de todo el mundo entre agosto de 2015 y febrero de 2016 debido a las fuertes caídas en las bolsas chinas, en especial en la de Shanghai. En este periodo se produjeron grandes ajustes en las cotizaciones debido a que los inversores advirtieron una sobrevaloración en los precios de las acciones. De hecho, uno de los sectores más desequilibrados de la economía china sigue siendo el sistema financiero, por tres razones: la proliferación de entidades bancarias y no bancarias y de productos de inversión, que dificultan la correcta supervisión financiera; la fuerte subida de precios de los activos (capital, bonos, inmuebles ${ }^{3}$ ) en un contexto de búsqueda de rentabilidad y valoración inadecuada del riesgo4; y fuertes salidas de capitales en 2015 y $2016^{5}$, en vista de las menores expectativas de crecimiento y la pérdida de rentabilidad de activos en moneda local (yuan o renminbi).

El otro caso llamativo son las empresas públicas chinas, muchas de las cuales han aprovechado los abultados subsidios públicos ${ }^{6}$ para operar con unas estructuras de costes abultadas y competir de forma ventajosa en los mercados internacionales. Precisamente la caída de la demanda internacional de muchos productos ha puesto el foco de todo el mundo en estas empresas, a las que se les exige reducir la sobrecapacidad en productos como el acero o el aluminio. Además, China cada vez

\footnotetext{
3 En Pekín y Shanghai, los precios de la vivienda nueva en 2016 subieron un 28,9 por 100 y un 34,8 por 100 , respectivamente. Algunos Gobiernos locales están limitando la especulación, y desde marzo de 2013 está vigente un impuesto del 20 por 100 sobre las plusvalías por la venta de viviendas.

4 El mercado bursátil chino se caracteriza por un elevado número de inversores individuales con pocos conocimientos financieros y de grandes empresas públicas (las empresas privadas no suelen cotizar en bolsa).

5 Desde noviembre de 2016 es necesario obtener la aprobación de la Administración estatal de cambio de divisas para ciertas transacciones con el exterior.

6 Según el FMI, estas empresas reciben un subsidio equivalente al 3 por 100 del PIB chino.
}

tiene menos argumentos para mantener algunas de estas empresas «zombis», que cuentan con unos beneficios y rentabilidades muy por debajo de las empresas privadas.

Es en este contexto en el que hay que entender la política económica de China. Lejos de aplicar un ajuste rápido de los desequilibrios (por ejemplo, reconociendo activos deteriorados, recortando drásticamente subsidios o asumiendo las nuevas valoraciones de los activos), el Gobierno chino ha implementado una estrategia a corto plazo y otra a medio y largo plazo. A corto plazo, el objetivo ha sido mantener el crecimiento económico y evitar las tensiones sociales internas. Por ejemplo, interviniendo en los mercados de divisas para limitar la fluctuación del tipo de cambio, restringiendo las operaciones bursátiles en caso de superar una determinada volatilidad o limitando las salidas de capitales. A largo plazo, el Gobierno ha diseñado una estrategia orientada a pasar de un modelo basado en el crecimiento del crédito y la industria a otro más basado en el consumo y los servicios. Este proceso quedó plasmado en el XIII Plan Quinquenal (2016-2020), que tiene tres grandes objetivos: dar prioridad al desarrollo económico, profundizar en las reformas estructurales (por ejemplo, reestructuración industrial, liberalización de algunos sectores) y acelerar la transformación del patrón de crecimiento (hacia un crecimiento basado en la innovación, respetuoso con el medio ambiente y más abierto al exterior).

\section{Estrategia de China en el ámbito bilateral}

\subsection{Estados Unidos y América Latina}

La llegada del presidente Trump a la Casa Blanca, en enero de 2017 , ha supuesto un $\triangleright$ 
cambio importante en las relaciones económicas entre los dos países. No en vano, gran parte del discurso del nuevo presidente de EEUU se basó en criticar la política comercial china, a la que acusa de generar enormes déficits comerciales en EEUU por la política de fuertes subsidios y competencia desleal. China es el primer proveedor de mercancías del país norteamericano, con una cuota de mercado del 21,2 por 100 en 2016. Destacan las importaciones de maquinaria eléctrica (129.000 millones de dólares), muebles (29.000 millones de dólares), juguetes y equipamiento deportivo $(24.000 \mathrm{mi}-$ llones de dólares), y calzado (15.000 millones de dólares).

Por su parte, China es el tercer cliente de EEUU, con una cuota de solo el 8 por 100. Destacan las exportaciones de semillas y soja (15.000 millones de dólares), aviones (15.000 millones de dólares) y maquinaria eléctrica (12.000 millones de dólares).

Sin embargo, un análisis más detallado muestra que las relaciones entre los dos países no son tan desequilibradas: EEUU tiene un superávit de servicios de 37.000 millones de dólares con China y existe una importante presencia de empresas norteamericanas en el país asiático, especialmente en sectores como las manufacturas y comercio al por mayor. Además, en los últimos diez años (20062016), la tasa de cobertura de EEUU respecto a China ha aumentado siete puntos porcentuales. Teniendo en cuenta estos hechos y los beneficios del comercio, el impacto de una eventual guerra comercial sería muy perjudicial para ambos países.

Además, a China se le está abriendo una oportunidad con el cambio de paradigma en la política comercial estadounidense. Incluso resulta llamativo ver, en los últimos meses, al presidente $\mathrm{Xi}$ Jinping defendiendo a ultranza la globalización y el sistema de comercio multilateral. Por ejemplo, el anuncio de Estados Unidos el 23 de febrero de 2017 de no ratificar el Acuerdo Transpacífico de Asociación ${ }^{7}$ (TPP, por sus siglas en inglés) fue una gran noticia para el gigante asiático, que veía en este bloque una amenaza para el dominio de las relaciones comerciales en la región. La Administración Obama siempre contempló este acuerdo como un instrumento clave para el denominado "giro asiático», con el objetivo de aumentar la presencia de EEUU en una de las zonas económicas más dinámicas del mundo (Asia-Pacífico) y, al mismo tiempo, evitar que China desempeñara un papel protagonista en dicha zona (China no participa en el TPP). Cada vez más, los países de la región empiezan a ver con entusiasmo la iniciativa de la Asociación Regional Económica Comprensiva (RCEP, por sus siglas en inglés) propuesta por China, que uniría los diez países de la ASEAN con los seis países que gozan de acuerdos de libre comercio con este bloque. Además, esta nueva iniciativa contempla menores estándares en alimentación, seguridad, empleo y divisas, y no impone restricciones en las empresas públicas chinas.

Más allá de su área de dominio natural, China ha interpretado la llegada de Trump como una oportunidad para conquistar nuevos mercados, no solo geográficos (como África o América Latina), sino también sectoriales. En efecto, los requerimientos de China respecto al resto del mundo han cambiado. Ya no busca mercados para abastecerse de materias primas baratas y exportar bienes manufacturados, sino alianzas de alto contenido tecnológico e intelectual. China es consciente de $\triangleright$

7 Acuerdo de libre comercio entre 12 países: Estados Unidos, Canadá, México, Perú, Chile, Australia, Nueva Zelanda, Japón, Singapur, Malasia, Vietnam y Brunéi. 
que debe desarrollar lazos fuertes con socios extranjeros, dejando que estos accedan al mercado interno pero que a cambio les faciliten derechos de propiedad intelectual. Y, de momento, ha conseguido algunos avances: los gastos de inversión en I+D han pasado del 0,7 por 100 en 1991 al 2,1 por 100 en 2015; los bienes de capital y productos de alta tecnología representan ya el 25 por 100 de las exportaciones chinas, y los productores chinos controlan entre el 15 y el 30 por 100 de los mercados globales para equipos de telecomunicaciones, turbinas eólicas y sistemas de ferrocarril de alta velocidad. En 2015 China lanzó la iniciativa «Made in China 2025» con el objetivo de transformar el país en un centro de manufacturas mundial innovador y medioambientalmente responsable. De hecho, China está desarrollando hubs de innovación y emprendimiento en Shenzen (donde ya operan grandes compañías innovadoras como Huawei, Xiaomi y DJI) y en el Parque de Ciencias de Zhonguancun, en Pekín. Todavía es difícil hablar de un éxito de este programa y un posible dominio chino en este ámbito con respecto a Estados Unidos en un futuro cercano, fundamentalmente porque para que el modelo tenga éxito debe existir una larga cadena institucional, social y legal que apoye la innovación y el desarrollo de nuevos productos. Por ejemplo, una adecuada protección de la propiedad intelectual (los delitos relacionados con los derechos de propiedad intelectual siguen siendo numerosos y afectan también a las empresas nacionales y a la política de independencia tecnológica que sigue el Gobierno), programas de grado o posgrado de calidad sobre áreas de alta tecnología y un flujo de información abierto a través de publicaciones especializadas. Un ejemplo de todo esto es que 10 de las 13 empresas que operan en China con un gasto en I+D superior al
6 por 100 de los ingresos son extranjeras. China gasta más de 220.000 millones de dólares en importar semiconductores y 21.000 millones de dólares en royalties para el uso de tecnologías de propiedad extranjera.

Además, todavía la mitad de las inversiones chinas se centran en campos petrolíferos y minería, y solo en los últimos años ha empezado a «escalar» en inversiones de alto valor añadido.

Desde un punto de vista geográfico, China ha sido consistente con su internacionalización más tardía que la de los países occidentales, adoptando una política de mayor riesgo. Así, la mitad de sus inversiones se sitúan en países en desarrollo en Asia, África, América Latina y Oriente Medio. Por ejemplo, China es el mayor inversor en países como Afganistán, Angola y Ecuador, y es hoy el mayor socio comercial de Brasil y Chile, por delante de EEUU, y ha firmado acuerdos de libre comercio con países como Perú. De esta forma, China ha desplazado a Japón como primer socio asiático del continente sudamericano. Desde el punto de vista de la inversión, China es uno de los principales inversores en países como Argentina, Brasil y Perú. Ahora el crecimiento de América Latina está mucho más influido por los vaivenes de la economía china. Se puede decir que en el caso de América Latina han coincidido dos factores: por un lado, las necesidades financieras y la abundancia de materias primas de los países latinoamericanos; y por otro, un exceso de liquidez inversora china y la búsqueda de materias primas. Sin embargo, la relación está siendo cada vez más asimétrica, en la medida en que China exporta productos con contenido más tecnológico y de alto valor añadido, mientras América Latina sigue exportando materias primas.

Por el contrario, en los países desarrollados la estrategia de inversión está siendo adquirir $\triangleright$ 
compañías tecnológicas de segundo plano, infravaloradas o en situación financiera complicada.

\subsection{Unión Europea}

Como ocurre en Estados Unidos, los productos chinos han invadido el mercado europeo, especialmente desde la adhesión de China a la Organización Mundial del Comercio (OMC) en 2001. Hoy, la Unión Europea sufre un déficit comercial de 175.000 millones de euros, de forma que las exportaciones europeas solo cubren el 50 por 100 de las importaciones chinas (en 2016 se exportaron productos por valor de 170.000 millones de euros, frente a importaciones de 345.000 millones de euros). Por productos, las principales exportaciones a China corresponden, por este orden, a automóviles y componentes de automoción (21,3 por 100 del total de exportaciones), productos farmacéuticos (4,4 por 100), material eléctrico (4,1 por $100)$ y aeronaves $(4,1$ por 100$)$. En cuanto a los productos importados de China por la Unión Europea, destacan los equipos de telecomunicaciones (12,4 por 100 del total), productos informáticos (10,6 por 100), textiles (5,2 por 100) y maquinaria agrícola (3,9 por 100).

Si observamos la tendencia de los últimos diez años, vemos que este déficit comercial es persistente y que no ha bajado de los 100.0000 millones de euros. Además, en los últimos años este déficit ha aumentado considerablemente (43.000 millones de euros del 2014 a 2015). La Unión Europea es el socio comercial más importante de China, y China es el segundo socio comercial mundial de la UE, por detrás de Estados Unidos ${ }^{8}$.

8 No obstante, esto podría cambiar si se consolida la salida de Reino Unido de la UE, ya que los países de la ASEAN ocuparían el primer lugar como socios comerciales de China.
Precisamente por este motivo, por la presencia cada vez más notable de las empresas chinas en sectores estratégicos europeos y por las presiones antiglobalización de los sectores más castigados por la pasada crisis económica y financiera, algunos países de la UE han aumentado la presión para defenderse de lo que se consideran prácticas comerciales injustas (dumping, subvenciones prohibidas) y amenazas a su seguridad nacional y a sectores estratégicos. Esta presión se ha hecho todavía más notable desde que China pasara a considerarse economía de mercado desde diciembre de 2016, conforme a lo acordado en su protocolo de adhesión a la OMC (2001).

Las complejas relaciones entre ambos bloques se hacen en la actualidad más difíciles por varios motivos. Por un lado, porque la UE está inmersa en las negociaciones de la salida de Reino Unido de la Unión Europea (brexit); por otro, porque el cambio de actitud de Estados Unidos ha provocado que ahora China y la UE sean aliados «por accidente» en asuntos que hasta hace poco nadie habría imaginado. Por ejemplo, en la lucha contra el cambio climático. Una vez ratificado el Acuerdo de París, y tras anunciar Estados Unidos su salida del mismo en junio de este año, China y la UE han mostrado en diversos foros su compromiso de darle continuidad. $Y$ ambos bloques están amenazados por la imposición de medidas proteccionistas por parte de Estados Unidos (por ejemplo, en el sector del acero), lo que justifica la defensa del libre comercio, aunque con matices, por ambos bloques en los recientes foros internacionales.

A pesar de las intensas relaciones económicas y comerciales, la UE y China no tienen firmado ningún acuerdo de libre comercio, pero desde el año 2008 se reúnen en el marco del Diálogo de Alto Nivel Económico y $\triangleright$ 
Comercial. El principal punto de discusión en la actualidad es el acuerdo de inversión, que lleva negociándose desde el año 2013 y que se consideraría la antesala de un eventual acuerdo de libre comercio. En las negociaciones de un acuerdo de inversión, una de las grandes preocupaciones de Europa son los subsidios (implícitos o explícitos) que reciben las empresas chinas de titularidad estatal. Por un lado, porque se perciben como competencia desleal en el mercado europeo, y, por otro, porque en ocasiones estas inversiones se realizan en sectores estratégicos, en los que cabe adivinar un interés no meramente económico, amenazando el orden público y la seguridad nacional. Por esta razón, en la actualidad se está debatiendo una propuesta para restringir la inversión extranjera en sectores estratégicos de la Unión Europea.

Además, la UE está modernizando los instrumentos de defensa comercial y la legislación antidumping y antisubvenciones. Se trata de una respuesta contra las prácticas comerciales desleales, pero que tiene como fondo la consideración automática de China como economía de mercado desde diciembre de 2016 y los problemas de sobrecapacidad en algunos sectores. Los elementos clave de esta reforma son dos: aplicar una nueva metodología en los sectores o países en los que se compruebe que existe dumping y reformar la regla del derecho inferior (Lesser Duty Rule) ${ }^{9}$.

Europa siempre ha reclamado a China una mayor apertura de su mercado a las empresas extranjeras ${ }^{10}$. De hecho, si nos atenemos a las relaciones bilaterales de inversión, se observa

\footnotetext{
9 Hasta ahora, la UE ha limitado las medidas de defensa comercial ante casos de dumping al daño efectivamente sufrido por la industria europea. La idea de la reforma es exceptuar la aplicación de esta regla en casos tasados de distorsiones en algunos mercados.

10 De hecho, el índice de restricción de acceso al mercado de la OCDE sitúa a China como uno de los países del mundo con más restricciones en este aspecto.
}

que éstas no se corresponden en absoluto con los flujos de comercio. En 2015, el stock de inversión de la UE en China ascendía a 168.000 millones de euros (288.000 millones de euros si incluimos Hong Kong), mientras que el stock de inversión de China continental a la UE alcanzó solo 35.000 millones de euros (115.000 millones de euros incluyendo Hong Kong). En todo caso, el objetivo de las inversiones europeas en China ha ido cambiando con el tiempo: en un principio (hasta 2005) se relacionaban con los bajos costes laborales y de suelo, además de una regulación medioambiental muy poco exigente; entre 2005 y 2012, la inversión se dirigió sobre todo al mercado doméstico, y desde 2012 han ido ganando peso los servicios. Por su parte, la inversión china en la UE busca fundamentalmente acceder a mercados, marcas y tecnología, muchas veces con objeto de replicarlos en su propio mercado.

No obstante, cabe esperar que estas relaciones de inversiones aumenten en los próximos tiempos, de acuerdo con la tendencia de los últimos años y la progresiva liberalización y apertura de China al mercado global. De hecho, China ha modificado su Catálogo para guiar la inversión de industrias extranjeras, reduciendo las restricciones de 93 en 2015 a 63 , aparte de la lista negativa de sectores en donde está excluida la inversión extranjera (televisión, prensa, finanzas, telecomunicaciones, etcétera). Parece que fuera de estos sectores las autoridades chinas quieren avanzar hacia un sistema de acceso a posteriori, de forma que se pasa de un control previo de acceso al mercado a una supervisión después de entrar. Además, el Gobierno chino ha indicado que quiere ofrecer a los inversores extranjeros el mismo trato que a los nacionales cuando se están planteando hacer una inversión. 


\section{Estrategia de China en el ámbito multilateral}

El ascenso de China como gran potencia económica mundial ha levantado dudas sobre sus verdaderas intenciones respecto al orden económico establecido, en el sentido de si realmente China quiere ostentar una supremacía económica (y política) en el mundo y alterar las reglas de juego (cambio de equilibrios en las instituciones multilaterales, nuevas prioridades de organismos internacionales, etcétera). De una manera simple y breve, podemos responder que no. Lo que parece que China está buscando es dominar, eso sí, la evolución de su región (Este asiático) y, a nivel global, tener la capacidad y libertad de seguir su propio camino, manteniendo el objetivo de avanzar en el crecimiento económico y el desarrollo sin detrimento del necesario equilibrio social interno.

\subsection{Dominio regional}

Desde la llegada de Xi Jinping al poder en 2012 se ha producido un cambio en la estrategia de China con respecto a sus vecinos asiáticos. En efecto, desde finales de los años setenta China adoptó una actitud de amistad y cooperación con sus vecinos, fundamentalmente por necesidad económica: China se encontraba económicamente por debajo no solo de Japón y Corea del Sur, sino también de otros países asiáticos como Malasia, Singapur, Tailandia e Indonesia. Esta realidad ha cambiado. En 2014, el Fondo Monetario Internacional (FMI) anunció que China era la economía más grande del mundo medida en términos de paridad de poder adquisitivo. Este cambio de actitud se ha visto en muchos frentes políticos (por ejemplo, construcción de islas artificiales en el
Mar de China o restringiendo en la pasada primavera operaciones de empresas surcoreanas cuando Corea del Sur desplegó un sistema americano antimisiles).

Pero el ejemplo más evidente de la nueva política china en la región es la Iniciativa de la Nueva Ruta de la Seda (Belt and Road Initiative, OBOR). La primera vez que se propuso fue a través del presidente Xi Jinping en 2013, con la idea de conectar por tierra y mar las rutas de comercio, inversión, finanzas, política y los intercambios entre personas de Asia, África y Europa. Es decir, desarrollar las infraestructuras para impulsar y reforzar la conectividad de China con sus socios más importantes. Esta iniciativa puede entenderse como una forma de contrarrestar la creación de una zona de libre comercio liderada por EEUU con algunos países del este asiático y de América (TPP). Pero también se puede interpretar como un intento de China en dar salida a sus excedentes de producción, ya que durante la crisis han aflorado muchas empresas con sobrecapacidad de producción. Además, el desarrollo de infraestructuras en el interior del país puede acelerar el crecimiento y la apertura de las regiones más aisladas, algunas de las cuales sufren tensiones o conflictos étnicos (provincia de Xinjiang). Nuevamente se introduce el objetivo del equilibrio y la estabilidad interna en las grandes estrategias del país.

El pistoletazo de salida de esta iniciativa se produjo en la Cumbre OBOR, en mayo de 2017 en Pekín, con la participación de numerosas delegaciones a nivel de jefes de Estado y de Gobierno (España participó en esta Cumbre). Aunque China ha comprometido una gran cantidad de recursos financieros en proyectos, los instrumentos financieros básicos son:

- Fondo de la Ruta de la Seda (Silk Road Fund). Es un nuevo fondo soberano $D$ 
dotado de 40.000 millones de dólares. Sus principales accionistas son los grandes financiadores de la expansión de las empresas chinas por el mundo: el fondo soberano China Investment Corporation, el Banco de Desarrollo de China (CDB) y el Eximbank. Este fondo aporta capital a los proyectos para que capten financiación de otras entidades.

- Banco Asiático de Inversiones e Infraestructuras (BAII). Fue lanzado en 2014 y constituido en diciembre de 2015 con 100.000 millones de dólares de capital. Este banco, que cuenta en la actualidad con más de setenta miembros ${ }^{11}$, concede créditos, garantías y participaciones en proyectos de infraestructuras favorables para el desarrollo de Asia. La creación de esta institución estuvo rodeada de controversia, ya que algunos países occidentales (principalmente Estados Unidos) se mostraron en contra por el debilitamiento del sistema creado en Bretton Woods (en donde el sistema de decisión está caracterizado por el derecho de veto de Estados Unidos ${ }^{12}$ ) y la posible rebaja que iba a suponer en los estándares, sociales, laborales y de transparencia para financiar proyectos de infraestructuras. Tras la entrada de Reino Unido, fueron muchos los países que finalmente dieron el paso, convencidos de que suponía una oportunidad para sus empresas en esos mercados y que finalmente se iban a respetar unos mínimos estándares internacionales. En todo caso, China no

11 Fue firmado por 57 países, de los cuales 37 son regionales (Asia, Rusia y Oceanía) y 20 no regionales (de los cuales 14 son de la UE). EI capital suscrito por los países regionales (incluido Rusia) y Oceanía no puede ser inferior al 75 por 100 del capital suscrito total.

12 Precisamente, parece que uno de los desencadenantes de esta "ofensiva» fue el bloqueo de la decimocuarta reforma de cuotas del FMI durante muchos años por parte de Estados Unidos. ha abandonado las instituciones tradicionales: sigue siendo el tercer financiador más importante del Banco Asiático de Desarrollo, y cada vez tiene más peso en el Banco Mundial ${ }^{13}$.

También hay que mencionar otras iniciativas regionales, como la Asociación Regional Económica Comprensiva, compuesta por los diez miembros de la ASEAN y países con los que esta guarda acuerdos de libre comercio (Australia, China, India, Japón, Corea del Sur y Nueva Zelanda). Representan casi la mitad de la población mundial, cerca del 30 por 100 del PIB global y un 25 por 100 de las exportaciones mundiales. El objetivo es crear un área de libre comercio entre estos países mediante un acuerdo moderno, comprensivo, de calidad y mutuamente beneficioso, cubriendo el comercio de bienes, servicios, inversiones, cooperación económica y técnica, propiedad intelectual, competencia, comercio electrónico, resolución de disputas y otros asuntos.

Otro caso es la Cumbre de Asia Oriental (EAS, por sus siglas en inglés), de la que China es principal organizadora y miembro fundador (2013), que está dedicada a garantizar la paz y la estabilidad de las regiones de Asia. Además, China participa en el Foro de Cooperación Económica AsiaPacífico (APEC) desde 1991 y colabora con la Asociación de Naciones del Sudeste Asiático (ASEAN) en su formato ASEAN + 3 (Japón, China y Corea del Sur).

Aunque no es eminentemente regional, China ha jugado un papel muy importante en la cooperación e integración entre los grandes países emergentes, como contrapeso a $D$

13 Además, China ha superado al Banco Mundial en préstamos bilaterales a países en desarrollo, aunque con menor rigor en lo que se refiere a buena gobernanza y transparencia. 
EEUU y, en menor medida, a Europa. Este grupo de países, que se conoce con el acrónimo BRICS (Brasil, Rusia, India, China y Sudáfrica), en 2014 decidió crear una institución financiera para invertir en infraestructuras y proyectos de desarrollo en países emergentes y en desarrollo. Se trata del Nuevo Banco de Desarrollo, que nació formalmente en 2015 con un capital inicial de 100.000 millones de dólares, con una participación y con suscripciones de capital iguales entre todos sus miembros.

\subsection{Instituciones y foros multilaterales}

China es miembro fundador de algunas de las más importantes instituciones globales, incluyendo Naciones Unidas, el Fondo Monetario Internacional y el Banco Mundial. En otras instituciones se incorporó más tarde, como a la OMC, donde ingresó en 2001 después de unas largas negociaciones, y con un proceso de transición de quince años. China también es miembro del G20, pero no del G7 ni de la OCDE. Aunque las autoridades chinas han reafirmado su compromiso con el orden internacional, en los últimos años se han involucrado en el establecimiento de nuevas reglas e iniciativas. En Naciones Unidas, China ha triplicado su presupuesto y desplegado miles de soldados en misiones de paz. En el ámbito del cambio climático, China ha sido uno de los principales impulsores del Acuerdo de París de 2015. Si bien nunca formó parte del Protocolo de Kyoto, la industrialización desordenada y los efectos de la contaminación en muchas ciudades asiáticas han provocado un cambio de rumbo, hasta el punto de que han mantenido el compromiso en este ámbito a pesar de la anunciada salida de Estados Unidos del Acuerdo de París.

\subsection{EI G20}

China forma parte del G20 desde su creación en 1999. Se trata de un grupo formado por Estados Unidos, China, India, Alemania, Francia, Italia, Reino Unido, Argentina, Brasil, Arabia Saudí, Japón, Australia, Canadá, Indonesia, la Unión Europea, Rusia, Sudáfrica, Turquía, Corea del Sur y México ${ }^{14}$. El objetivo ha sido siempre la coordinación de las políticas económicas para promover la estabilidad financiera y el crecimiento económico global. El valor añadido de este foro de coordinación económica es que reúne a los principales países desarrollados y emergentes ${ }^{15}$ en pie de igualdad, y que permite la discusión y la cooperación en múltiples ámbitos que afectan a la economía global. Otra particularidad importante es que las decisiones se toman por consenso, impulsando de esta manera las discusiones de temas en los organismos internacionales en que participan. No obstante, el problema que presenta este foro ( $y$, en general, la gran mayoría de organismos multilaterales) es que no cuenta con mecanismos sancionadores o de coacción que obliguen a implementar los compromisos asumidos.

Aunque las decisiones se toman por consenso, el papel de China en el G20 es muy relevante. Al mismo tiempo, China ha aprovechado este foro para mejorar su imagen, incorporar nuevos temas en las discusiones, e impulsar avances en los temas que más le preocupan. Esto ocurrió sobre todo durante la presidencia china del G20 en 2016. China demostró una gran capacidad de liderazgo, operativo y de medios en $\triangleright$

\footnotetext{
14 España tiene un estatus especial de invitado permanente. Además, cada año la presidencia del G20 invita a una serie de países en función de criterios de vecindad, de diversificación geográfica y de nivel de desarrollo.

15 No obstante, el PIB absoluto no es el único factor que determina la inclusión de un país en este foro, ya que en su momento se atendió también al equilibrio geográfico y al nivel de desarrollo de los países.
} 
la organización de las reuniones de trabajo durante todo ese año, especialmente para la celebración de la Cumbre de Jefes de Estado y de Gobierno en Hangzhou. Como ejemplo, cabe decir que parte de la ciudad fue desalojada durante esos días y se limitaron las emisiones de las fábricas cercanas a la ciudad para reducir la contaminación ${ }^{16}$. Desde el punto de vista del contenido, China supo organizar unos temas de discusión muy en línea con su política económica y su transición a una economía de consumo y servicios, en la que el conocimiento y las tecnologías de la información y las comunicaciones tengan un mayor papel.

En 2016 se consolidó la importancia de una nueva área de trabajo relacionada con la innovación, aprobándose un Plan sobre Crecimiento Innovador (Blueprint on Innovative Growth). La idea era aumentar la capacidad de crecimiento de la economía en base a cuatro pilares: innovación, nueva revolución industrial, economía digital y reformas estructurales ${ }^{17}$. En definitiva, frente a los últimos compromisos y prioridades del G20, China planteó un enfoque más a medio y largo plazo para modificar el patrón de crecimiento y aumentar la productividad y la capacidad de crecimiento potencial. Es importante recordar que uno de los grandes objetivos del XIII Plan Quinquenal vigente (2016-2020) es precisamente acelerar la transformación del patrón de crecimiento en un país que hasta ahora se ha basado en una industrialización acelerada, intensiva en mano de obra, y en la exportación de manufacturas, y que debe adaptarse rápidamente a la nueva era digital y a los cambios que exige su propia senda de crecimiento.

\footnotetext{
16 Algunas fuentes no oficiales situaron el gasto para la Cumbre de Hangzhou en 24.000 millones de dólares.

17 Los líderes aprobaron un Plan de Acción en Innovación, otro en Nueva Revolución Industrial y una Iniciativa sobre Desarrollo y Cooperación en Economía Digital.
}

China está manteniendo un perfil alto en algunos temas económicos de discusión del G20:

1. Economía global y mercados financieros: como ya se ha comentado, China es, junto al gran bloque de países emergentes y en desarrollo, un firme defensor de un reparto más equilibrado de las cuotas de poder (voto) en las instituciones financieras multilaterales, especialmente en el Fondo Monetario Internacional. El principal argumento es que los países emergentes y en desarrollo no están suficientemente representados en estas instituciones. Así, China está abogando por la decimoquinta revisión de cuotas en el FMI, que debe incluir una nueva fórmula para el cálculo de las cuotas. Además, apoya los trabajos en financiación verde y los esfuerzos de colaboración en fiscalidad internacional (aplicación del paquete anti BEPS, transparencia e intercambio de información, fiscalidad de los nuevos modelos de negocio y, sobre todo, mayor asistencia a países en desarrollo para ayudarles a cumplir los estándares internacionales de intercambio de información). En 2015, China consiguió que el FMI incluyera su moneda en la cesta que componen los derechos especiales de giro (DEG) ${ }^{18}$. Este hecho tuvo una gran importancia para China, pues supone un reconocimiento de la importancia de la divisa china en el sistema financiero internacional y de que su valor obedece a criterios de mercado. Actualmente, lo que defiende China es un mayor uso de estos derechos $\triangleright$

18 Se hizo efectivo en octubre de 2016. 
especiales de giro en el ámbito internacional. Por ejemplo, promoviendo la emisión de bonos del sector privado en DEG, nuevas emisiones de DEG, o incentivando la contabilidad de los organismos internacionales en DEG. Aunque China ha creado instituciones financieras multilaterales, no pretende romper el esquema vigente y suele apoyar las iniciativas para aumentar la colaboración entre ellas o la catalización de inversión privada.

2. En comercio e inversión, China está asumiendo junto con la Unión Europea un mayor papel de liderazgo a favor del comercio internacional y la globalización ante el nuevo tono proteccionista de Trump. En cierta medida, esto se corresponde con el modelo de crecimiento que ha tenido China en los últimos treinta años y con su consideración como economía de mercado en la OMC desde diciembre de 2016. No obstante, China defiende que el comercio debe conducir a un crecimiento más inclusivo, y ello exige eliminar referencias a un marco de reglas equilibrado y a un comercio justo, algo que cada vez más reclaman los países desarrollados. China mantiene numerosos contenciosos comerciales con Estados Unidos y con la Unión Europea por la aplicación de medidas proteccionistas (medidas antidumping, antisubvención y salvaguardia), aunque también sufre reclamaciones en la OMC por parte de estos países. Parece que China justificaría la aplicación de políticas distorsionantes e incompatibles con las reglas de la OMC para fomentar el crecimiento y la inclusión, rechazando la aplicación de medidas proteccionistas contra productos y empresas chinas. En los últimos años, este enfrentamiento se ha centrado especialmente en los esfuerzos por atajar el exceso de producción en sectores como el acero. De hecho, el G20 acordó la creación de un Foro Global con secretariado de la OMC para aumentar la comunicación y la colaboración, así como mejorar el funcionamiento de los mecanismos de mercado y el ajuste de la sobrecapacidad. Este Foro está muy basado precisamente en la cooperación y el intercambio de información, algo en lo que China no está siendo especialmente proactiva (por ejemplo, propone siempre respuestas colectivas y es reticente a un esquema de intercambio de información sobre capacidad instalada frecuente que permita la rápida reacción ante excesos de producción). Lo cierto es que hasta ahora no se ha conseguido acordar un paquete de medidas efectivas para acabar con el problema de la sobrecapacidad, y es de esperar que China alargue el proceso de decisión mientras va ajustando la sobrecapacidad de su industria de manera autónoma y controlada.

3. Innovación y digitalización: los intereses de China en este ámbito son diversos. Por un lado, es una firme defensora de la formación digital y la incorporación de las tecnologías y las comunicaciones a la industria. En este sentido, le interesa el flujo de información y know-how para adquirir conocimiento y facilitar su cambio de patrón de crecimiento. Pero esto choca con la política china de control de información para fines de seguridad nacional (ciberseguridad), privacidad y estabilidad interna. Por eso, China no $\triangleright$ 
aboga por un verdadero flujo libre de información. Además, no están de acuerdo en acordar estándares internacionales para la producción digitalizada.

4. Energía y cambio climático: como se ha mencionado, China ha reiterado su compromiso con el Acuerdo de París y está comprometida con la reducción de gases de efecto invernadero. Aunque se trata del mayor emisor mundial de dióxido de carbono (30 por 100 del total) y todavía el 60 por 100 de la energía consumida en el país procede del carbón, China se ha comprometido a reducir las emisiones en el futuro. El principal motor fue el compromiso que sellaron China y Estados Unidos en 2014, y que a la postre arrastró a todos los grandes contaminantes, como India, a la firma del Acuerdo de París en diciembre de 2015 (en la Conferencia de las Partes, COP21). Las declaraciones de los líderes chinos y los planes puestos en marcha para fomentar las energías limpias (fundamentalmente energía eólica y solar) no deben ocultar los intereses internos para que China no cumpla con lo prometido. De hecho, la salida de EEUU del Acuerdo de París supone un argumento que las grandes empresas estatales chinas emplearán para no alterar el mix energético, al menos tan rápido. Además, el coste de las energías renovables y el impacto en el empleo (muy dependiente todavía del carbón y el acero) han arrojado dudas sobre la verdadera credibilidad del compromiso de China con el cambio climático y las energías limpias.

\section{Conclusiones}

Aunque el fuerte crecimiento económico de China ha sido contemplado por algunos como una amenaza para el orden económico internacional vigente desde Bretton Woods, sus prioridades son dos: a nivel regional, ostentar una hegemonía económica, política y militar que asegure su estabilidad interna y su prestigio internacional; y a nivel mundial, seguir aprovechando el orden económico e institucional existente (del que tanto se ha beneficiado hasta ahora), mientras se asegura la adquisición del necesario know-how para ayudar en su transición a una economía más innovadora y basada en los servicios y el consumo. De su capacidad para tejer alianzas en un entorno multipolar como el actual dependerá el éxito de su objetivo.

\section{Bibliografía}

[1] BARQUERO CABRERO, J. D. y ROCAFORT NICOLAU, A. (2010). La economía china: un reto para Europa. Gerona: Furtwangen.

[2] BROWN, K. (2017). The Critical Transition: China's Priorities for 2021.

[3] DELAGE, F. (2011). «Bajo la sombra de China». Economía Exterior, n.ำ 56.

[4] ESTEBAN, M. (2017). El efecto Trump en las relaciones UE-China. Real Instituto Elcano.

[5] FEIGENBAUM, E.A. (2016). China and the World.

[6] FENBY, J. (2017). Can China rule the world?

[7] FONTAINE, R. y RAPP-HOOPER, M. (2016). «The China Syndrome». The National Interes, n.. 143, pp. $10-18$.

[8] GHEMAWAT, P.y HOUT, T. (2016). Can China's Companies Conquer the World?

[9] PASTOR, A. (2011). «El regreso de China». Economía Exterior, n. .56 .

[10] PETTIS, M. (2017). China should not liberalize the economy.

[11] SUMMERS, T. (2017). EU-China economic relations to 2025: building a common future. 
